\title{
Semiaffine Spaces
}

\author{
Hendrik Van Maldeghem* \\ Department of Pure Mathematics and Computer Algebra \\ Ghent University, Krijgslaan 281-S22, B-9000 Ghent \\ hvm@cage.ugent.be
}

Submitted: Jul 6, 2008; Accepted: Jan 26, 2009; Published: Feb 4, 2009

Mathematics Subject Classification: 51A45

\begin{abstract}
In this paper we improve on a result of Beutelspacher, De Vito \& Lo Re, who characterized in 1995 finite semiaffine spaces by means of transversals and a condition on weak parallelism. Basically, we show that one can delete that condition completely. Moreover, we extend the result to the infinite case, showing that every plane of a planar space with at least two planes and such that all planes are semiaffine, comes from a (Desarguesian) projective plane by deleting either a line and all of its points, a line and all but one of its points, a point, or nothing.
\end{abstract}

\section{Introduction}

In [2], Beutelspacher, De Vito and Lo Re prove that linear spaces in which disjoint lines are either "weakly parallel", or admit, through any given point outside the two lines, at most one transversal, and which satisfy a rather strong condition on these weakly parallel lines, are planar spaces all of whose planes are semiaffine. They then use an unpublished result of Teirlinck and the classification of semiaffine planes due to Dembowski \& Kuiper [3] to determine all finite such linear spaces. In the present paper we show that one can delete the condition on the parallel lines, and moreover we bypass the classification of finite semiaffine planes and proceed applying Teirlinck's theorem when there are at least two planes, so that also all infinite such linear spaces are classified.

More exactly, we show (but postpone the precise definitions to the next section):

First Main Result. Let $\mathcal{S}=(\mathcal{P}, \mathcal{L})$ be a linear space such that through every point outside two given disjoint lines either there is at most one transversal, or every line

\footnotetext{
${ }^{*}$ Partially supported by the Fund of Scientific Research - Flanders
} 
meeting one of the given disjoint lines meets the other. If every line contains at least three points, then $\mathcal{S}$ is a planar space in which every plane is semiaffine.

Second Main Result Let $\mathcal{S}=(\mathcal{P}, \mathcal{L})$ be a planar space with at least two planes, such that every line contains at least four points and all planes of which are semiaffine. Then every plane is obtained from a projective plane by deleting either nothing, a single point, a line with all but one of its points, or a line with all its points.

Main Corollary Let $\mathcal{S}=(\mathcal{P}, \mathcal{L})$ be a linear space all lines of which contain at least four points. Then the following are equivalent.

(SAS1) Through every point $x$ outside two given disjoint lines $L_{1}, L_{2}$ either there passes at most one transversal, or every line through $x$ meeting $L_{1} \cup L_{2}$ in at least one point meets $L_{1} \cup L_{2}$ in exactly two points. Also, the first possibility really occurs.

$(\mathrm{SAS} 2) \mathcal{S}$ is a planar space with at least two planes such that every plane is a semiaffine plane.

$(\mathrm{SAS} 3) \mathcal{S}$ is an affino-projective semiaffine space (and hence arises from a unique projective space by deleting a set $S$ of points in a hyperplane with the property that, whenever a line meets $S$ in at least two points, then it meets $S$ in either all its points, or all but one of its points).

This result motivates the definition of a semiaffine space as either a semiaffine plane, or a planar space satisfying the condition in (SAS2). It then follows that (SAS1) is a characterization of semiaffine spaces with line size at least three (if one deletes the restriction that the first possibility in (SAS1) must really occur), and that (SAS3) provides a classification of semiaffine spaces with line size at least four, and containing at least two planes. We will give more details concerning (SAS3) below.

Note that our main results are tokens of general and classical geometric occurrences. In particular, the First Main Result is a kind of counterpart for linear spaces of the characterization of polar spaces by Beukenhout \& Shult [1] by means of the so-called one-or-all axiom. For linear spaces, a one-or-all axiom cannot involve only a point and one line, but a natural way to state such an axiom is to use one point and two disjoint lines. Here, the possibility of 'zero' must also be considered (if one adds this possibility for linear spaces, then one obtains the definition of a so-called gamma space, a notion which plays a central role in the theory of (Grassmannian) point-line geometries from spherical buildings). Hence, the First Main Result characterizes semiaffine spaces by a none-one-or-all axiom. Moreover, if one excludes the possibility of parallel lines, i.e., if one assumes that in (SAS1) only the first possibility can occur, then the condition simplifies to Pasch's famous axiom. So, the First Main Result can also be seen as a generalization of the Veblen \& Young [5] characterization for projective space by including the affine spaces and spaces that lie in between an affine and a projective space, and which we called semiaffine spaces. Thirdly, the Second Main Result expresses the idea that, once put in a higher dimensional object, objects of rank two tend to be tame. For instance, 
projective planes inside projective spaces of dimension at least three are automatically Desarguesian. More generally, generalized polygons in irreducible spherical buildings of rank at least three are Moufang. In our case, semiaffine planes of semiaffine spaces with at least two planes arise automatically from Desarguesian projective planes by deleting either nothing, a point, an affine line and all of its points, or a projective line and all of its points. Note that there are free constructions of semiaffine planes that do not arise from any projective plane in the above mentioned way, see [3].

In our proof, we will use the results of [2]. In fact, we reduce the situation to the hypotheses therein. Also, for our Second Main Result, we assume that lines have infinite size (the finite case following directly from [3]), although our proof almost completely works under the assumption of at least four points per line. We did not try to make it work completely under this assumption (in view of [3]), but this might not at all be a difficult exercise.

\section{Preliminaries}

A linear space $\mathcal{S}=(\mathcal{P}, \mathcal{L})$ consists of a set $\mathcal{P}$ of points and a family $\mathcal{L}$ of subsets of $\mathcal{P}$, called lines, such that each pair of distinct points $x, y$ is contained in a unique line, sometimes denoted by $x y$. The prominent examples of linear spaces are the projective spaces, which arise from vector spaces by taking for point set the the set of one-dimensional subspaces of a vector space $V$, and as lines the sets of one-dimensional subspaces contained in a two-dimensional subspace. Let, for a given linear space $(\mathcal{P}, \mathcal{L})$, the set $\mathcal{P}^{*}$ denote the set of all pencils, where a pencil is the set of lines containing a given point. A projective plane is a linear space $(\mathcal{P}, \mathcal{L})$ such that all lines have size at least three, and such that $\left(\mathcal{L}, \mathcal{P}^{*}\right)$ is also a linear space. An affine plane is a linear space in which the parallel axiom for lines holds: every point is contained in exactly one line disjoint from or equal to a given line. A semiaffine plane is a linear space in which a slightly weaker form of the parallel axiom holds: every point is contained in at most one line disjoint from or equal to a given line. In [3], Dembowski \& Kuiper show that every finite semiaffine plane is constructed from a projective plane by deleting either nothing, a point, a line and all of its points, or a line and all but one of its points. Moreover, they show that the finiteness hypothesis is essential by constructing counterexamples in the infinite case.

Now let $\mathcal{S}=(\mathcal{P}, \mathcal{L})$ be a linear space. A transversal of two disjoint lines $L_{1}, L_{2}$ is a line meeting $L_{1} \cup L_{2}$ in two points. Consider the following none-one-or-all axiom:

(NOA) For every point $x$ and every pair of disjoint lines $L_{1}, L_{2}$ not containing $x$, exactly one of the following possibilities occurs:

(i) there is no transversal of $L_{1}, L_{2}$ containing $x$;

(ii) there is exactly one transversal of $L_{1}, L_{2}$ containing $x$;

(iii) every line through $x$ meeting $L_{1}$ or $L_{2}$ is a transversal of $L_{1}, L_{2}$. 
Let $L_{1}, L_{2}$ be two disjoint lines of $\mathcal{S}$. If, for some $x$, every line through $x$ meeting $L_{1}$ or $L_{2}$ is a transversal of $L_{1}, L_{2}$, then Beutelspacher, De Vito \& Lo Re [2] say that $L_{1}$ and $L_{2}$ are parallel, and the point $x$ is called a witness for the parallelity of $L_{1}, L_{2}$. We shall also adopt this terminology, which allows to rephrase Axiom (NOA) as if some point $x$ is contained in at least two transversals of some pair of disjoint lines $L_{1}, L_{2}$ not containing $x$, then $L_{1}$ and $L_{2}$ are parallel and $x$ is a witness for their parallelity.

A subspace $\mathcal{S}^{\prime}=\left(\mathcal{P}^{\prime}, \mathcal{L}^{\prime}\right)$ of $\mathcal{S}$ consists of a subset $\mathcal{P}^{\prime} \subseteq \mathcal{P}$ of the point set and a subset $\mathcal{L}^{\prime} \subseteq \mathcal{L}$ of the line set such that the union of $\mathcal{L}^{\prime}$ equals $\mathcal{P}^{\prime}$, and such that $\mathcal{S}^{\prime}$ is a linear space. Given a family $\mathcal{E}$ of subspaces, we say that $\mathcal{S}=(\mathcal{P}, \mathcal{L}, \mathcal{E})$ is a planar space if every triple of noncollinear points is contained in exactly one member of $\mathcal{E}$. The elements of $\mathcal{E}$ are then called planes. Concerning terminology, we say that the unique plane containing three given noncollinear points is spanned by these points. Similarly, a plane can also be spanned by a line and a point not on that line, or by two distinct intersecting lines.

The prototype of planar spaces are the affine and projective spaces of dimension at least three. One can, of course, also consider spaces that somehow "lie between affine and projective spaces". More exactly, we consider the following family of planar spaces.

Let $\mathcal{S}$ be a projective space, viewed as a linear (or planar) space, and let $H$ be a hyperplane (i.e., a subspace intersecting every line nontrivially). If we remove all points and lines (and planes) of $H$ from $\mathcal{S}$, then we obtain an affine linear (planar) space $\mathcal{S}_{H}$. Also, if $\mathcal{S}^{\prime}$ is a projective subspace of $\mathcal{S}$, then the intersection $\mathcal{S}_{H}^{\prime}$ with $\mathcal{S}_{H}$ yields an affine subspace of $\mathcal{S}_{H}$, which we call, by abuse of notation, also an affine subspace of $\mathcal{S}$. The subspace $\mathcal{S}^{\prime}$ will be called the projective completion of $\mathcal{S}_{H}^{\prime}$. We can now consider a family $\mathfrak{F}$ of affine subspaces of $\mathcal{S}$ with the following properties.

$(\mathrm{AP} 1) \mathcal{S}_{H} \in \mathfrak{F}$

(AP2) The point sets of any two members of $\mathfrak{F}$ are disjoint.

(AP3) If $\mathcal{S}_{1}, \mathcal{S}_{2} \in \mathfrak{F}$, then, for some $i \in\{1,2\}$, the point set of $\mathcal{S}_{i}$ is contained in the point set of the projective completion of $\mathcal{S}_{3-i}$.

If we take the union of the point sets of $\mathfrak{F}$, and endow it with all lines (and planes) induced from $\mathcal{S}$, then we denote the resulting linear (planar) space by $\mathcal{S}_{\mathfrak{F}}$. Condition (AP3) clearly ensures that $\mathcal{S}_{\mathfrak{F}}$ is a semiaffine space, and therefore we call it an affino-projective semiaffine space. This construction provides all examples described between parentheses in (SAS3) above, and conversely. This can be easily checked.

We now briefly review some known results. We start with the main point of this paper.

Fact 1 (Beutelspacher, De Vito, Lo $\operatorname{Re}[2])$ Let $\mathcal{S}=(\mathcal{P}, \mathcal{L})$ be a linear space such that through every point outside two given disjoint but nonparallel lines there is at most one transversal. Then $\mathcal{S}$ is a semiaffine space, granted that every point on every transversal of two parallel lines (but not contained in these lines) is a witness for their parallelity. 
Note that the conditions in the previous fact immediately imply that $\mathcal{S}$ satisfies Condition (NOA). But if (NOA) is satisfied, then, in order to obtain the conditions of Fact 1, one has to require that, $\left(^{*}\right)$ if for a point $x$ and two disjoint lines $L_{1}, L_{1}$ not containing $x$, there are at least two transversals of $L_{1}, L_{2}$ through $x$, this is also true for every other choice of $x$ on any transversal of $L_{1}, L_{2}$ (as long as $x \notin L_{1} \cup L_{2}$ ). Our main aim is to entirely delete this condition $(*)$, i.e., we will derive $\left(^{*}\right)$ from (NOA).

Fact 2 (Teirlinck [4]) Let $\mathcal{S}=(\mathcal{P}, \mathcal{L}, \mathcal{E})$ be a planar space all planes of which are affinoprojective semiaffine, and such that every line has at least four points. Then $\mathcal{S}$ is an affino-projective semiaffine space.

Fact 3 (Dembowski \& Kuiper [3]) Every finite semiaffine plane is affino-projective.

Corollary 4 (Beutelspacher, De Vito, Lo Re [2]) Let $\mathcal{S}=(\mathcal{P}, \mathcal{L})$ be a finite linear space such that each line contains at least four points and such that through every point outside two given disjoint but nonparallel lines there is at most one transversal. Then $\mathcal{S}$ is an affino-projective semiaffine space, granted that every point on every transversal of two parallel lines (but not contained in these lines) is a witness for their parallelity.

The aim of the present paper is to considerably weaken the conditions of Fact 1 by virtually deleting the last condition (i.e., we rephrase as done above and delete Condition $(*)$ ), and to remove the finiteness condition in Corollary 4 . The latter will be done by proving directly that every plane of a semiaffine space containing at least two planes is affino-projective, granted there are infinitely many points per line. Of course, the case where $\mathcal{S}$ is a semiaffine plane cannot be included since there are counterexamples, see [3]. Hence we have the following theorem.

Theorem 5 A linear space in which every line carries at least four points and which satisfies Condition (NOA) is either a line, a semiaffine plane, or an affino-projective semi-affine space.

It is clear that Theorem 5 follows from our First and Second Main Results.

Note that Fact 1 is also true for linear spaces with arbitrary line size, in particular when there are lines of size two. However, this cannot be true for our First Main Result as the following counterexample shows. Let $\mathcal{S}$ consist of a point $\infty$ and two disjoint families of points $\mathcal{P}^{\prime}$ and $\mathcal{P}^{\prime \prime}$. The lines are all pairs of points where one point belongs to $\mathcal{P}^{\prime}$ and the other to $\mathcal{P}^{\prime \prime}$, together with the sets $\mathcal{P}^{\prime} \cup\{\infty\}$ and $\mathcal{P}^{\prime \prime} \cup\{\infty\}$. Then $\mathcal{S}$ satisfies the conditions of our First Main Result (except for the line sizes of course), but not of Fact 1 . Moreover, it is not a semiaffine plane. So we do need the condition on the size of the lines in our First Main Result. 


\section{Proofs}

\subsection{First Main Result}

By Fact 1, it suffices to show that, for two parallel lines $L_{1}$ and $L_{2}$, and any transversal $M$, every point $x$ on $M \backslash\left(L_{1} \cup L_{2}\right)$ is a witness for the parallelity of $L_{1}$ and $L_{2}$. We prove a few lemmas.

Throughout, we assume that $L_{1}$ and $L_{2}$ are two given parallel lines, with witness $p$.

Lemma 6 Every line parallel to $L_{1}$ with witness $p$ is also parallel to $L_{2}$ with witness $p$.

Proof Suppose, by way of contradiction, that a line $L^{\prime}$ intersects $L_{2}$ in a point $a$, and that $L^{\prime}$ is parallel with $L_{1}$ with witness $p$. Let $a^{\prime}$ be the intersection of $L_{1}$ and ap. Pick $b$ on $L^{\prime}$, with $b \neq a$. Pick $b^{\prime}$ on $L_{1}$, with $b^{\prime} \neq a^{\prime}$ and $b^{\prime} \notin p b$. Note that $p b$ meets $L_{1}$. Consider the line $b b^{\prime}$.

- Assume $b b^{\prime}$ meets $L_{2}$. Then the two lines $b b^{\prime}$ and $b p$ through $b$ meet both of $L_{1}$ and $L_{2}$, hence, by (NOA), $b$ is a witness for the parallelity of $L_{1}$ and $L_{2}$. Consequently ba, which equals $L^{\prime}$, must meet $L_{1}$, too, a contradiction to our assumption.

- Now assume that $b b^{\prime}$ is disjoint from $L_{2}$, and hence is parallel with it with witness $p$ (since $p b$ meets $L_{2}$ because it meets $L_{1}$, and $p b^{\prime}$ meets $L_{2}$ since it meets $L_{1}$, and $L_{1}$ and $L_{2}$ are parallel with witness $p$ ). This implies that $p a$ meets $b b^{\prime}$, say in the point $c$.

Pick $d$ on $L^{\prime}, d \notin\{a, b\}$. Pick $e$ on $L_{2}, e \neq a$, and $e \notin p d$ (for instance, to fix the ideas, we can choose $e$ on the line $p b$ ). If de met $L_{1}$, then, since $p d$ meets $L_{1}$ and hence $L_{2}$, we see that $d$ would be a witness for the parallelity of $L_{1}$ and $L_{2}$, implying $d a=L^{\prime}$ meets $L_{1}$, a contradiction. Hence de is disjoint from $L_{1}$. But both of $p d$ and pe meet $L_{1}$; hence $p$ is a witness for the parallelity of $L_{1}$ and $d e$. It follows that $p a$ meets $d e$. Now, if $d e$ and $b b^{\prime}$ were disjoint, then they would be parallel with witness $a$, as $L^{\prime}$ and $a p$ both meet both of $d e$ and $b b^{\prime}$. This would imply that also $L_{2}$ meets $b b^{\prime}$, a contradiction. So $e d$ and $b b^{\prime}$ meet in a point $f$.

Since $e d$ and $L_{1}$ are parallel with witness $p$, the line $f p$ meets $L_{1}$ and hence also $L^{\prime}$. So both lines $f p$ and $f b$ meet both of $L^{\prime}$ and $L_{1}$, hence the latter are parallel with witness $f$, implying $f d$ meets $L_{1}$, the final contradiction.

Hence $L^{\prime}$ meets $L_{1}$ and the lemma is proved.

We can already conclude the following.

Corollary 7 Every point not on $L_{1} \cup L_{2}$ on a transversal of $L_{1}$ and $L_{2}$ that contains $p$, is a witness for the parallelity of $L_{1}$ and $L_{2}$. 
Proof If $x$ is such a point, then by the previous lemma, every line through $x$ meeting $L_{1}$ meets $L_{2}$ and vice versa.

We now prove that every transversal of $L_{1}$ and $L_{2}$ contains a witness for the parallelity of $L_{1}$ and $L_{2}$.

Lemma 8 Every transversal $M$ of $L_{1}$ and $L_{2}$ contains a witness for the parallelity of $L_{1}$ and $L_{2}$.

Proof Set $x_{i}:=L_{i} \cap M, i=1,2$, and set $y_{i}=p x_{3-i} \cap L_{i}, i=1,2$. Let $z_{1}$ be a point on $L_{1}, z_{1} \notin\left\{x_{1}, y_{1}\right\}$. If $p z_{1}$ meets $M$, then the result follows from Corollary 7. So we may assume that $p z_{1}$ and $M$ are disjoint, in which case they are parallel with witness $y_{2}$ (noting that $p z_{1}$ meets $L_{2}$ ). Consequently $y_{2} z_{1}$ meets $M$ in some point $w$. But now clearly $w$ is a witness for the parallelity of $L_{1}$ and $L_{2}$ and belongs to $M$.

Combining Corollary 7 with Lemma 8, the First Main Result follows from Fact 1.

\subsection{Second Main Result}

Now we assume that $\mathcal{S}$ is a semiaffine planar space, and we may assume that each line has infinitely many points. We forget about the definition of parallelity previously given, and call now two lines $L, M$ parallel, in symbols $L \| M$, if they are coplanar and disjoint or equal. Our first aim is to prove that parallelism is an equivalence relation.

Lemma 9 Parallelism in a semiaffine space $\mathcal{S}$ is an equivalence relation.

Proof Suppose $L_{1}\|L\| L_{2}$. We clearly may assume that $L_{1} \neq L_{2} \neq L \neq L_{1}$. Let $\pi_{i}$ be the plane containing $L$ and $L_{i}, i=1,2$. Note that $L_{1}$ and $L_{2}$ are disjoint as otherwise $\pi_{1}=\pi_{2}$ and so $L_{1}=L_{2}$ by the axioms of semiaffine planes. Pick points $x_{i} \in L_{i}, i=1,2$, and let $x$ be an arbitrary point on $L$. It is easy to see that there exists a line $M$ meeting $x x_{1} \cup x x_{2} \cup x_{1} x_{2}$ in three distinct points $y, y_{1}, y_{2}$, with $y \in x_{1} x_{2}, y_{i} \in x x_{i}, i=1,2$. Choose a point $x^{\prime} \in L, x^{\prime} \neq x$. In $\pi_{i}$, the line $x^{\prime} y_{i}$ meets $L_{i}$ in some point $x_{i}^{\prime}, i=1,2$ (otherwise there are two lines through $x^{\prime}$ in $\pi_{i}$ parallel to $L_{i}$ ). Consider the plane $\pi$ generated by $x^{\prime}, y_{1}, y_{2}$.

Suppose first that for some $i \in\{1,2\}$, the line $y x_{i}^{\prime}$ meets the line $x^{\prime} x_{3-i}^{\prime}$, say in the point $z$. Then the plane $\alpha$ generated by $y$ and $L_{i}$ contains $x_{3-i}$ and $z$, and so it meets the plane $\pi_{3-i}$ in the line $K:=x_{3-i} z$ (clearly, $x_{3-i} \neq z$ ). If $K$ met $L$, then the intersection point would belong to $\alpha$. Since also $L_{i}$ belongs to $\alpha$, this would imply that $\alpha$ coincides with $\pi_{i}$, contradicting $y \notin \pi_{i}$. Hence $K$ and $L$ are disjoint in $\pi_{3-i}$, implying that $K=L_{3-i}$. So $\alpha$ contains the disjoint lines $L_{1}$ and $L_{2}$; consequently $L_{1} \| L_{2}$.

Now suppose that $x_{1}^{\prime} y$ is disjoint from $x^{\prime} x_{2}^{\prime}$ and $x_{2}^{\prime} y$ is disjoint from $x^{\prime} x_{1}^{\prime}$. We can find $y_{0} \in y_{1} y_{2}, y_{1} \neq y_{0} \neq y_{2}$ and $x_{0} \in x_{1} x_{2}, x_{1} \neq x_{0} \neq x_{2}$, such that $x_{0} y_{0}$ contains $x$. Then 
$x_{i}^{\prime} y$ meets $x^{\prime} y_{0}$, say in the point $z_{i}, i=1,2$. The same argument as in the foregoing paragraph now shows that $z_{i} \neq x_{0}$ and $x_{0} z_{i}$ is parallel to $L$ in the plane $\pi_{0}$ spanned by $L$ and $x_{0}, i=1,2$; this already implies $x_{0} z_{1}=x_{0} z_{2}=: L_{0}$. Furthermore, as in the previous paragraph, $L_{1}\left\|L_{0}\right\| L_{2}$ and $L_{0}, L_{i}$ are contained in the plane $\alpha_{0}$ spanned by $y$ and $L_{0}$, for $i=1,2$. Hence $\alpha_{0}$ contains both $L_{1}$ and $L_{2}$ and the lemma is proved.

The next lemma shows that each parallel class of lines is either trivial or large, in the sense that, if the class is not trivial, each plane through every member of the parallel class contains a lot of other members.

Lemma 10 Let $L$ be a line of $\mathcal{S}$, and let $\pi_{1}, \pi_{2}$ be two distinct planes through $L$. If some line in $\pi_{1}$ is parallel to $L$, but distinct from $L$, then there is at most one point $x$ in $\pi_{2}$ not belonging to a line parallel to $L$.

Proof Suppose there are two points $x, y$ in $\pi_{2}$ with the property that no line in $\pi_{2}$ through $x$ or $y$ is parallel to $L$. Then the line $x y$ is certainly not parallel to $L$ and meets $L$ in some point $z$. Choose a second point $z^{\prime} \neq z$ on $L$. Let $L_{1} \neq L$ in $\pi_{1}$ be parallel to $L$ and choose two points $z_{1}, z_{1}^{\prime}$ on $L_{1}$, with $z_{1} \neq z_{1}^{\prime}$, in such a way that $z z_{1}$ and $z^{\prime} z_{1}^{\prime}$ meet in a point $t$. Choose a point $u$ on $x y, u \notin\{x, y, z\}$, such that the line $T:=t u$ is not parallel to either $x z_{1}$ or $y z_{1}$ (here we actually need at least six points on the line $x y$; in the finite case with at least four points per line, another argument should be included). It follows that $x z_{1}$ meets $T$ in some point $p_{x}$ and $y z_{1}$ meets $T$ in some point $p_{y}$.

Consider the plane $\alpha$ spanned by $T$ and $z^{\prime}$, which also contains $z_{1}^{\prime}$. One of the lines $p_{x} z_{1}^{\prime}$, $p_{y} z_{1}^{\prime}$ is not parallel to $u z^{\prime}$ in $\alpha$; say $p_{x} z_{1}^{\prime}$ meets $u z^{\prime}$ in a point $x^{\prime}$. Now the plane $\alpha_{x}$ spanned by $p_{x}$ and $L_{1}$ contains two points (namely, $x$ and $x^{\prime}$ ) of $\pi_{2}$, and hence it contains the line $x x^{\prime}$ of $\pi_{2}$. Clearly $x x^{\prime}$ is parallel to $L_{1}$, hence also to $L$, a contradiction.

It now follows that there are two types of lines in $\mathcal{S}$ : one type of lines, which we call projective, consists of those lines that have no parallel line; the other type, called affine, consists of lines that have parallel lines in every plane to which they belong.

Using this lemma, we can now use exactly the same proof as for Lemma 10 to show the following slightly stronger result.

Lemma 11 Let $L$ be an affine line in some plane $\pi_{2}$. If there exists a point $x$ in $\pi_{2}$ not contained in a line of $\pi_{2}$ parallel to $L$, then $x$ belongs to every projective line contained in $\pi_{2}$.

Proof Suppose $x \in \pi_{2}$ is not contained in a line of $\pi_{2}$ parallel to $L$, and suppose $x$ does not belong to a given projective line $L^{\prime}$ of $\pi_{2}$. Then, in the proof of Lemma 10, we can disregard $y$ and we can choose $z$ outside $L^{\prime}$, we can choose $z^{\prime}$ in $L \cap L^{\prime}$, and we can choose $u$ on $L^{\prime}$ (and $u \in\{x, z\}$, as required). Then, with the above notation, the line $p_{x} z_{1}^{\prime}$ always meets $z^{\prime} u$ in some point $x^{\prime}$ and $x x^{\prime}$ is parallel to $L$, a contradiction.

We now handle the case where a plane contains two intersecting affine lines. 
Lemma 12 Let $\pi$ be a plane of $\mathcal{S}$ and suppose that $\pi$ contains two intersecting affine lines $L_{1}, L_{2}$. Then either $\pi$ is an affine plane, or $\pi$ is an affino-projective semiaffine plane arising from an affine plane by adding just one point at infinity.

Proof Let $\pi, L_{1}, L_{2}$ be as described. Since we assume that $\mathcal{S}$ has at least two planes, there must be a point outside $\pi$, and hence there exists a line $K$ not belonging to $\pi$ but intersecting $\pi$ in $L_{1} \cap L_{2}=: x$. By Lemma 10, there is at most one point $x_{i}$ on $K$ such that the plane $\alpha_{i}$ spanned by $L_{i}$ and $K$ contains no line through that point parallel to $L_{i}$, $i=1,2$. Since lines have at least four points (in fact, we assume infinitely many), we find a point $x^{\prime}$ on $K$ and distinct lines $L_{1}^{\prime}, L_{2}^{\prime}$ parallel to $L_{1}, L_{2}$, respectively, and containing $x^{\prime}$. Denote the plane spanned by $L_{1}^{\prime}, L_{2}^{\prime}$ by $\pi^{\prime}$. Since clearly the lines $L_{1}^{\prime}$ and $L_{2}^{\prime}$ do not have any point in common with $\pi$ (as $K$ does not belong to $\pi$ and every triple of noncollinear points is contained in a unique plane), the planes $\pi$ and $\pi^{\prime}$ meet in at most one point (if they met in a line, then this line would meet at least one of $L_{1}^{\prime}$ or $L_{2}^{\prime}$, contradicting our observation just made). Hence there are two possibilities.

- Suppose $\pi \cap \pi^{\prime}=\emptyset$. Let $y_{i}$ be an arbitrary point on $L_{i}, i=1,2$, with $y_{i} \neq x$. Let $k$ be a point on $K$, with $x \neq k \neq x^{\prime}$. In the plane $\alpha_{i}$, the line $k y_{i}$ is not parallel to $L_{i}^{\prime}$, hence it meets it in a point $y_{i}^{\prime}, i=1,2$. The lines $y_{1} y_{2}$ and $y_{1}^{\prime} y_{2}^{\prime}$ belong to the same plane and are disjoint; so they are parallel. Hence $y_{1} y_{2}$ is an affine line. Given $z_{1}$ on $L_{1}$, with $z_{1} \neq x$, we can find a point $z_{1}^{\prime}$ on $L_{1}^{\prime}$, with $z_{1}^{\prime} \neq x^{\prime}$, such that $y_{1} z_{1}^{\prime} \cap K \neq \emptyset \neq z_{1} z_{1}^{\prime} \cap K$. Performing twice the construction above with $k$ substituted with $y_{1} z_{1}^{\prime} \cap K$ and $z_{1} z_{1}^{\prime} \cap K$, respectively (and the second time the roles of $\pi$ and $\pi^{\prime}$ interchanged), we see that there is a line in $\pi$ containing $z_{1}$ and parallel to $y_{1} y_{2}$. Replacing $L_{1}$ by a parallel line, we see that there is also a line parallel to $y_{1} y_{2}$ containing $x$. Similarly, we also see that all lines in $\pi$ must be affine. It now follows easily that through every point of $\pi$ there is a line parallel to any given line. Hence $\pi$ is an affine plane.

- Suppose now $\pi \cap \pi^{\prime}=\{p\}$, with $p \in \mathcal{P}$. Similarly as above, one shows that all lines of $\pi$ not containing $p$ are affine lines, and that for every point $q \neq p$, and every line $L$ of $\pi$, with $p \notin L$, there exists a line $L^{\prime} \subseteq \pi$, with $q \in L^{\prime} \| L$.

Suppose now, by way of contradiction, that there was an affine line $M$ in $\pi$ containing $p$. Then there would be a line $M^{*}$ parallel to $M$ inside $\pi$ not containing $p$. With the notation of the previous paragraphs, this would imply that $M^{*}$ is parallel to some line $M^{\prime}$ in $\pi^{\prime}$. By the transitivity of parallelism (see Lemma 9), this yields $M \| M^{\prime}$. But no plane contains both $M$ and $M^{\prime}$ as the plane generated by $M^{\prime}$ and $p \in M$ does not contain $M$, a contradiction.

Hence all lines in $\pi$ through $p$ are projective. Removing $p$ from $\pi$, we clearly obtain an affine plane.

The lemma is proved. 
From now on, we consider only planes $\pi$ having no intersecting affine lines, i.e., planes admitting only one parallel class of lines. Of course, if there is no affine line, then the plane is a projective plane, and we are done. Hence we may assume that there is at least one affine line $L$. Since all lines of $\pi$ meeting $L$ are projective, Lemma 11 implies that $\pi$ is the union of all lines of $\pi$ parallel to $L$. Adding a point at infinity corresponding with that parallel class of lines in the usual way, we see that $\pi$ arises from a projective plane by deleting one point and no lines.

Hence we have proved:

Theorem 13 In a semiaffine planar space with at least two planes, every plane is an affino-projective semiaffine plane.

Our Second Main Result now follows from Teirlinck's result Fact 2. However, a direct proof is also possible, and we sketch one here (but leave the details to the reader). We add to $\mathcal{S}$ a point at infinity for each parallel class of lines, and add that point to each line of that parallel class. We add lines at infinity, containing points at infinity and possibly ordinary points, corresponding to the lines at infinity of the semiaffine planes of $\mathcal{S}$ which arise from affine planes where at most one point at infinity is added. Hence we have completed every semiaffine plane to a projective plane. It is now trivial to check Pasch's axiom if all lines considered are contained in the completion of a semiaffine plane (belonging to $\mathcal{E}$ ). So we are reduced to check Pasch's axiom in the case where the elements are not contained in any member of $\mathcal{E}$, but are all "at infinity" of some planes. This case reduces readily to the "three-dimensional" case, i.e., to a subspace of $\mathcal{S}$ generated by two planes intersecting in a line. Using the structure of affino-projective semiaffine planes, the rest of the proof is a tedious but elementary exercise. So one obtains a projective space in which $\mathcal{S}$ is naturally embedded, and now the Second Main result is clear.

\section{References}

[1] F. Buekenhout \& E. E. Shult, On the foundations of polar geometry, Geom. Dedicata 3 (1974), 155-170.

[2] A. Beutelspacher, P. De Vito \& P. M. Lo Re, A characterization of linear spaces based on the number of transversals, Geom. Dedicata 54 (1995), 255-261.

[3] P. Dembowski, Semiaffine Ebenen, Arch. Math. 13 (1962), 120-131.

[4] L. Teirlinck, Combinatorial Structures, Ph.D.-thesis, Dept. Mathematics, Vrije Universiteit Brussel VUB, Brussels, 1974.

[5] O. Veblen \& J. W. Young, Projective Geometry, Blaisdell, New York, 1910. 